This item was submitted to Loughborough's Research Repository by the author.

Items in Figshare are protected by copyright, with all rights reserved, unless otherwise indicated.

\title{
Development of a volumetric projection technique for the digital evaluation of
} field of view

PLEASE CITE THE PUBLISHED VERSION

http://dx.doi.org/10.1080/00140139.2013.815805

PUBLISHER

(c) Taylor \& Francis

VERSION

AM (Accepted Manuscript)

LICENCE

CC BY-NC-ND 4.0

\section{REPOSITORY RECORD}

Marshall, Russell, Steve Summerskill, and Sharon Cook. 2019. "Development of a Volumetric Projection Technique for the Digital Evaluation of Field of View". figshare. https://hdl.handle.net/2134/12834. 
This item was submitted to Loughborough's Institutional Repository (https://dspace.lboro.ac.uk/) by the author and is made available under the following Creative Commons Licence conditions.

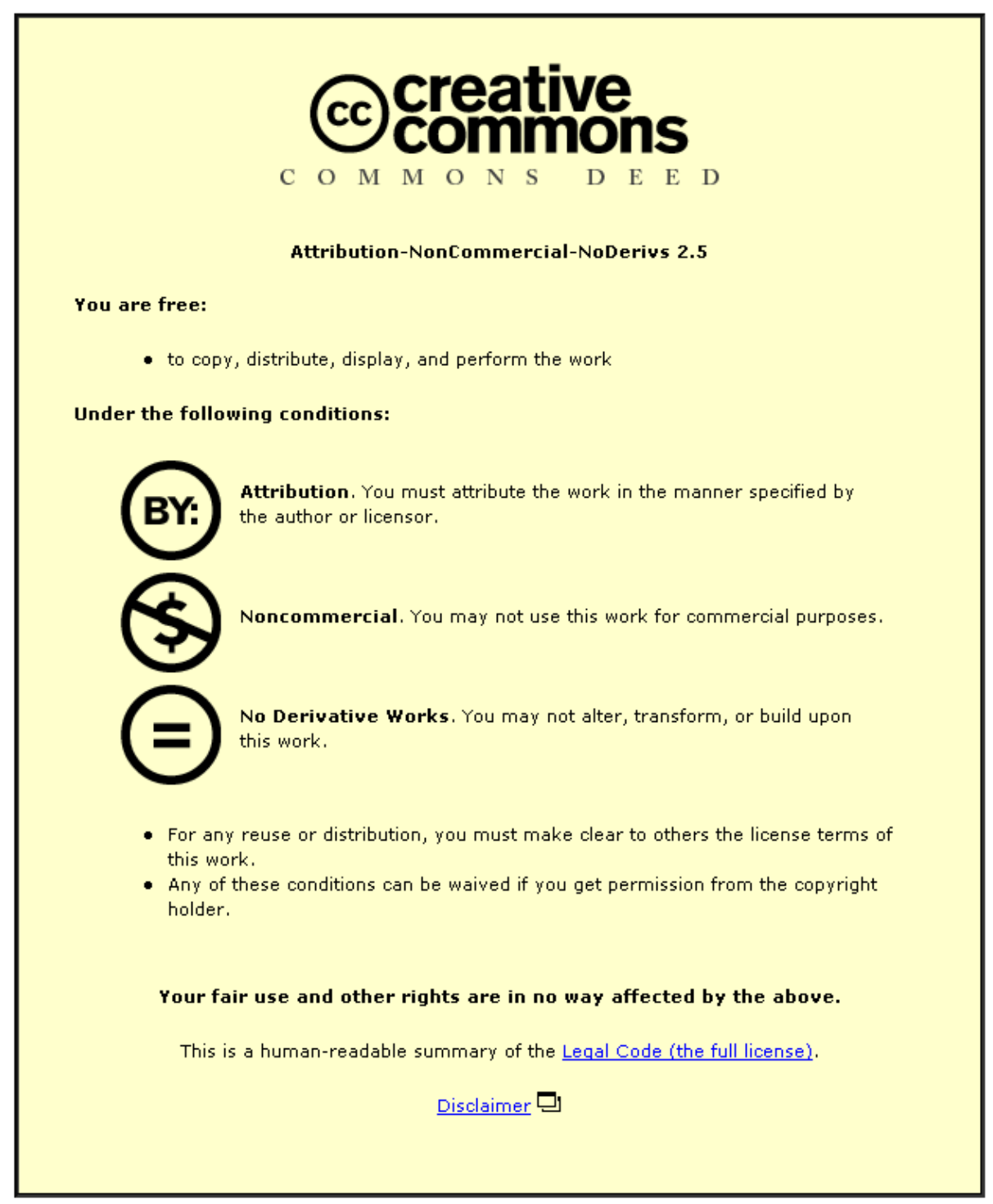

For the full text of this licence, please go to: http://creativecommons.org/licenses/by-nc-nd/2.5/ 
Development of a volumetric projection technique for the digital evaluation of field of view

Russell Marshall, Stephen Summerskill, Sharon Cook

Loughborough Design School, Loughborough University, Loughborough, LE11 3TU, $U K$

Email: R.Marshall@1boro.ac.uk

Published in Ergonomics (2013): Development of a volumetric projection technique for the digital evaluation of field of view, Ergonomics

http://dx.doi.org/10.1080/00140139.2013.815805 


\title{
Development of a volumetric projection technique for the digital evaluation of field of view
}

\author{
Current regulations for field of view requirements in road vehicles are defined by \\ $2 \mathrm{D}$ areas projected on the ground plane. This paper discusses the development of \\ a new software based volumetric field of view projection tool and its \\ implementation within an existing digital human modelling (DHM) system. In \\ addition, the exploitation of this new tool is highlighted through its use in a UK \\ Department for Transport (DfT) funded research project exploring the current \\ concerns with driver vision. Focusing specifically on rearwards visibility in \\ small and medium passenger vehicles the volumetric approach is shown to \\ provide a number of distinct advantages. The ability to explore multiple \\ projections of both direct vision (through windows) and indirect vision (through \\ mirrors) provides a greater understanding of the field of view environment \\ afforded to the driver whilst still maintaining compatibility with the $2 \mathrm{D}$ \\ projections of the regulatory standards.
}

Keywords: vision, field of view, digital human modelling, road vehicles

\section{Practitioner summary}

Field of view requirements for drivers of road vehicles are defined by simplified 2D areas projected onto the ground plane. However, driver vision is a complex 3D problem. This paper presents the development of a new software based 3D volumetric projection technique and its implementation in the evaluation of driver vision in small and medium sized passenger vehicles.

\section{Introduction}

Driver vision of the external environment from vehicles is a long standing issue and continues to be concern (Mansfield et al. 2008, Molinero et al. 2008, NHTSA 2008). One highly topical scenario includes injuries and fatalities to cyclists from collisions with large good vehicles (LGVs) in urban areas, where driver vision is a key potential 
causal factor in the occurrence of the accident (Cook et al. 2011). Drivers need to be able to view the driving environment as clearly as possible, preferably without obstruction and distortion. However, current vehicle design effectively ensures that there is likely to be some obstruction due to the solid structure of the vehicle occluding the driver's field of view. In addition, the driver should have a cognitive model of a full 360 degree field of view around the vehicle whilst also needing to attend to the primary driving task. As such, regulations specify the need for various field of view requirements for different vehicle types in an attempt to provide a view to the driver that will allow them to see what they need to see in any given situation. This is clearly a complex issue when all of the relevant variables are considered including the change in eye position due to the size of the driver and seat position and the specific design of the vehicle including windows, mirrors, pillars, head restraints etc. A review of the regulations that govern field of view in vehicles shows that the complexity of the situation has led to continuous amendment of the regulations. These amendments have taken place over many years, adapting to changes in vehicle design, advances in technology and the incremental advancement in understanding of the requirements of the driver, other road users, and the nature of the problem (e.g. Regulation 46 (UNECE Regulations 41-60 2012)). In addition considerable research has been targeted at the large number of factors surrounding driver vision including the impact of visual restrictions (Toet et al. 2008), comprehension of indirect views through mirrors (Hahnel and Hecht 2012), the effects of ageing (Gruber et al. 2013), the use of camera systems (Fitch et al. 2011), amongst others.

Typically two methods have been employed to evaluate vision from vehicles to assess the current situation in existing vehicles and driving tasks, or to inform the design of new vehicles. The first involves real world assessments, with a driver sat in an 
existing vehicle being asked to identify targets and other visual markers in the driving environment, directly, through window apertures, or indirectly through mirrors. This can be used to identify the visibility of real world targets such as pedestrians, cyclists, other vehicles and so on. Alternatively the field of view can be plotted on the ground to provide an area of visibility. These plots then provide a useful reference for the complete field of view afforded to the driver and allow for comparison between vehicles. Alternatively, field of view assessments are becoming increasingly the focus of digital techniques (Case et al. 1980, Krum 2009, Teizer et al. 2010). CAD models of vehicles can be combined with virtual humans in a digital human modelling (DHM) system and evaluations of vision performed in the digital environment. These digital evaluations are the equivalent of the real-world assessments with the ability to produce an image of the view from the virtual driver's eyes and also create projections of the field of view through windows and mirrors. The digital approach has many advantages through reduced cost and time, and the ability to evaluate rapidly the impact of changes in driver size, or adjustment of mirrors, for example. However, existing field of view projection techniques pose limitations on the assessments that can be performed and do not currently fully exploit the potential of the digital environment. The most significant limitation is the reliance on 2D area (e.g. ground plane) evaluations of field of view for what is essentially a $3 \mathrm{D}$ volumetric problem.

This paper presents the development of a new volumetric vision projection and assessment technique. The projection is implemented within an existing digital human modelling system and a case study is presented of its application to research in the transport realm. The research was performed by the Design Ergonomics Group and the User Centred Research Group at Loughborough Design School, Loughborough University and was commissioned by the UK Department for Transport (DfT) who had 
identified a number of concerns with vision from vehicles that they wished to explore. The DfT identified three specific areas for the research:

1. To fully understand the areas around Category $\mathrm{N}_{2}$ and $\mathrm{N}_{3}$ vehicles (LGVs with a maximum mass between 3.5 and 12 tonnes $\left(\mathrm{N}_{2}\right)$ and greater than 12 tonnes $\left.\left(\mathrm{N}_{3}\right)\right)$ that cannot be seen by the driver through direct or indirect vision.

2. To investigate and understand the field of view obscuration issues for Category $\mathrm{M}_{1}$ vehicles (vehicles designed and constructed for the carriage of passengers and comprising no more than eight seats in addition to the driver's seat) and their relationship with reported accident data. Investigations focused on the impact of A / B - pillar design, size and configuration.

3. To investigate and understand the rearward field of view obscuration issues for Category $\mathrm{M}_{1}$ (as defined above) and $\mathrm{M}_{2}$ vehicles (vehicles with more than 8 seats, not including the driver and a maximum mass of 5 tonnes or less).

The research focused specifically on the UK market and predominantly on right hand drive vehicles, however mirroring the situation in left hand drive vehicles makes the findings equally applicable for both configurations of vehicle and international markets. In addition, the impact of left hand drive vehicles on UK roads was also explicitly explored.

To investigate these issues the approach taken was to recreate a number of scenarios identified from UK accident data in which obscuration may have been a contributory factor. The scenarios would be evaluated using multiple vehicles selected using UK/EU sales statistics and to specifically evaluate vehicles with different configurations. The effect of different size drivers would also be considered.

To support these evaluations a volumetric field of view assessment method was developed that allows the volume of space visible through an aperture, or reflected 
through a mirror to be projected within the SAMMIE DHM (Porter et al. 2004)

environment. The method was designed to support multiple aperture / mirror

projections in order to describe a full 360 degree volumetric field of view assessment.

\section{Field of view evaluation techniques}

Driver field of view assessments in vehicles have been performed using a number of tools and methods in recent years (Tait and Southall 1998, Way and Reed 2003, Dodd 2009, Cook et al. 2011). One approach relies on the identification by the driver of visible targets placed outside of the vehicle. The driver is asked to report on visibility and / or recognition of the nature of the target. Often this form of analysis is accompanied by photographs approximating what the driver can see (Figure 1). Whilst this method is ideal for actually assessing, in real world conditions, what a driver can actually see and understand, it is difficult to quantify and to compare with other vehicles.

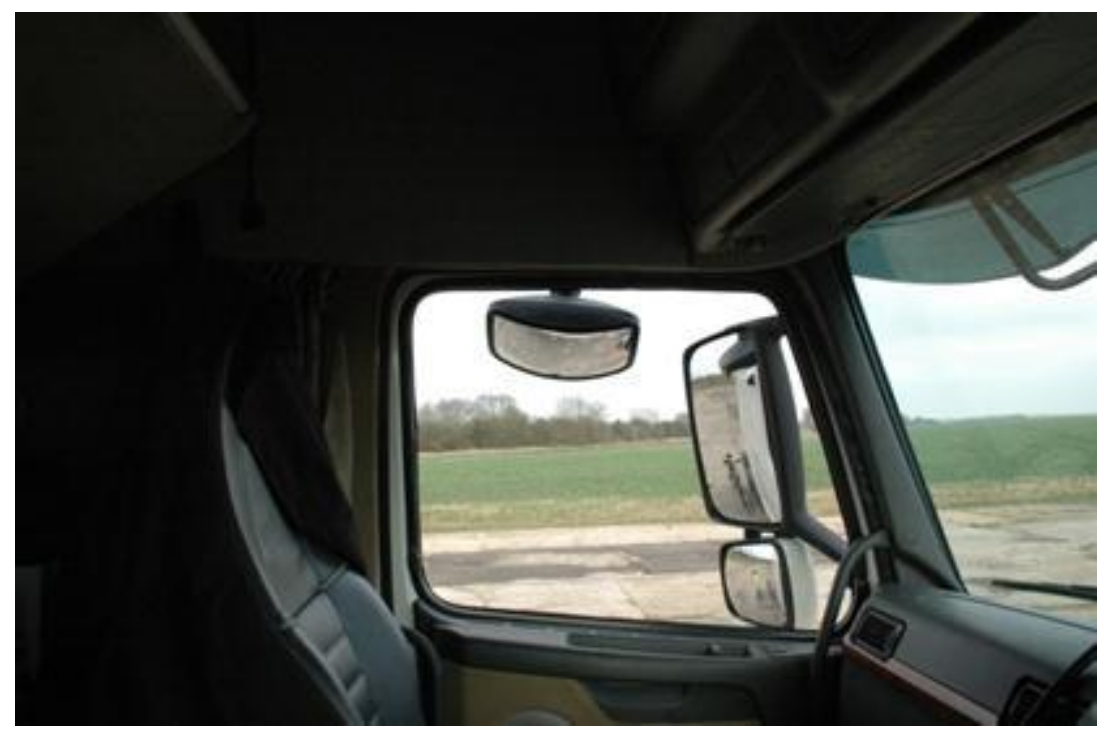

Figure 1. Image of the approximate view afforded to the driver of a VOLVO FH Category $\mathrm{N}_{3}$ vehicle

Another common approach to identify the field of view afforded to the driver is to use 2D mapping, in which the field of view through an aperture such as a window, or 
via a mirror, is projected onto the ground plane to plot the visible area (Figure 2). This approach is also employed in regulatory standards to describe the minimum field of view for vehicles of various types e.g. 2003/97/EC (2004) and UNECE Regulation 46 (2009) (Figure 3).

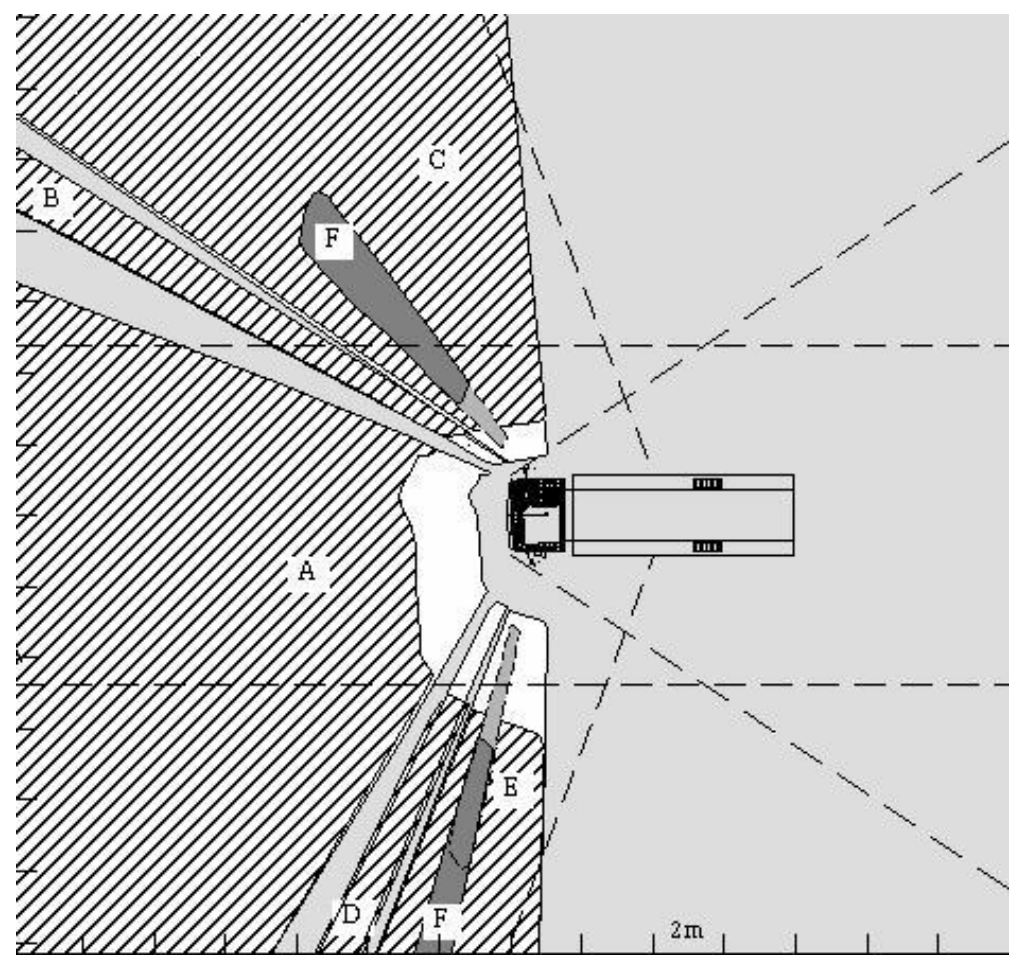

Figure 2. Example of a direct field of view plot (Leyland Daf truck) (From Tait and Southall, 1998)

The definition and evaluation of these areas has been performed using both realworld and virtual techniques. Real-world methods typically require direct observation by a driver and the positioning of target markers placed on the ground to identify the limits of view. However, light based techniques have also been employed whereby a lamp placed at the driver's eye point is projected from the vehicle and plotted on surfaces within a specially prepared room (Fosberry and Mills 1959). Virtual methods typically involve the projection of the aperture or mirror boundary onto the ground plane using a form of ray tracing. 


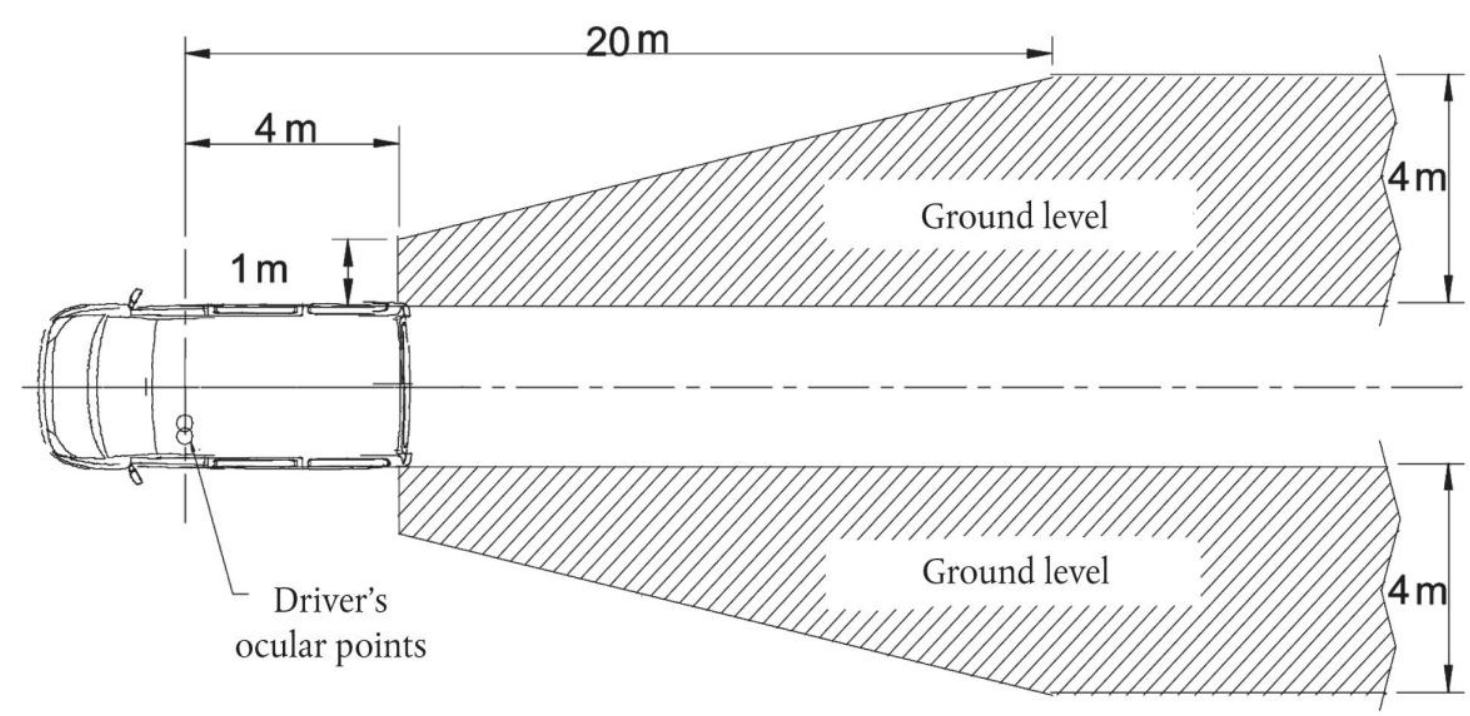

Figure 3. Minimum field of view specified in 2003/97/EC (2004) for Class III (exterior small) mirrors

\section{Limitations of current field of view specifications and evaluations}

Comparisons of real-world and virtual assessments have shown a high degree of correlation of both approaches (Cook et al. 2011, Reed et al. 2000). However, a fundamental issue in the use of these real-world or virtual assessments is the simplification of the field of view to a $2 \mathrm{D}$ area. In reality the field of view is a complex 3D volume, only part of which may intersect the ground plane. In addition multiple volumes of view may intersect in ways in which a $2 \mathrm{D}$ projection cannot capture.

Taking a 2D approach is not without its advantages. The use of $2 \mathrm{D}$ areas is beneficial in the definition of standards or regulations for minimum requirements. In addition, this simplified representation makes the specified field of view relatively straightforward to evaluate in the field, which is important if vehicles are to be assessed in their compliance with such standards. However, a 2D approach is also a considerable compromise and distortion of the real-world environment.

The use of the ground plane itself for the $2 \mathrm{D}$ area is questionable as most targets that a driver would wish to be aware of are not at this level, or rather the part of a target that you would wish to see is at a higher vertical level. Essentially this is an issue of 
recognition as much as visibility. For example a driver would want to see the torso, or head of a pedestrian, and not their feet, or the body of a car and not its tyres as this would ensure greater recognition of the visual target. The use of virtual techniques to create projections of the visible volume clearly illustrates the potential limitations with the $2 \mathrm{D}$ area approach. The areas defined in Figure 2 can be interpreted that the driver would be able to see that area on the ground plane and suggest that something in that area would also be clearly visible. Yet this is not as clear as it may first appear, as the volume of viewable space is not necessarily uniform in the vertical component. Thus a target positioned in the visible area on the ground plane may only stay visible for a small portion of its height, or a target positioned outside of the visible area may become visible part way up its height. Figure 4 shows the 3D volumetric projection of the field of view from the driver's side mirror of a 2010 model UK Volkswagen golf. A human is stood within the visible area as projected at the ground plane but the visible volume indicates that only their feet and part of their lower leg would be visible to the driver.

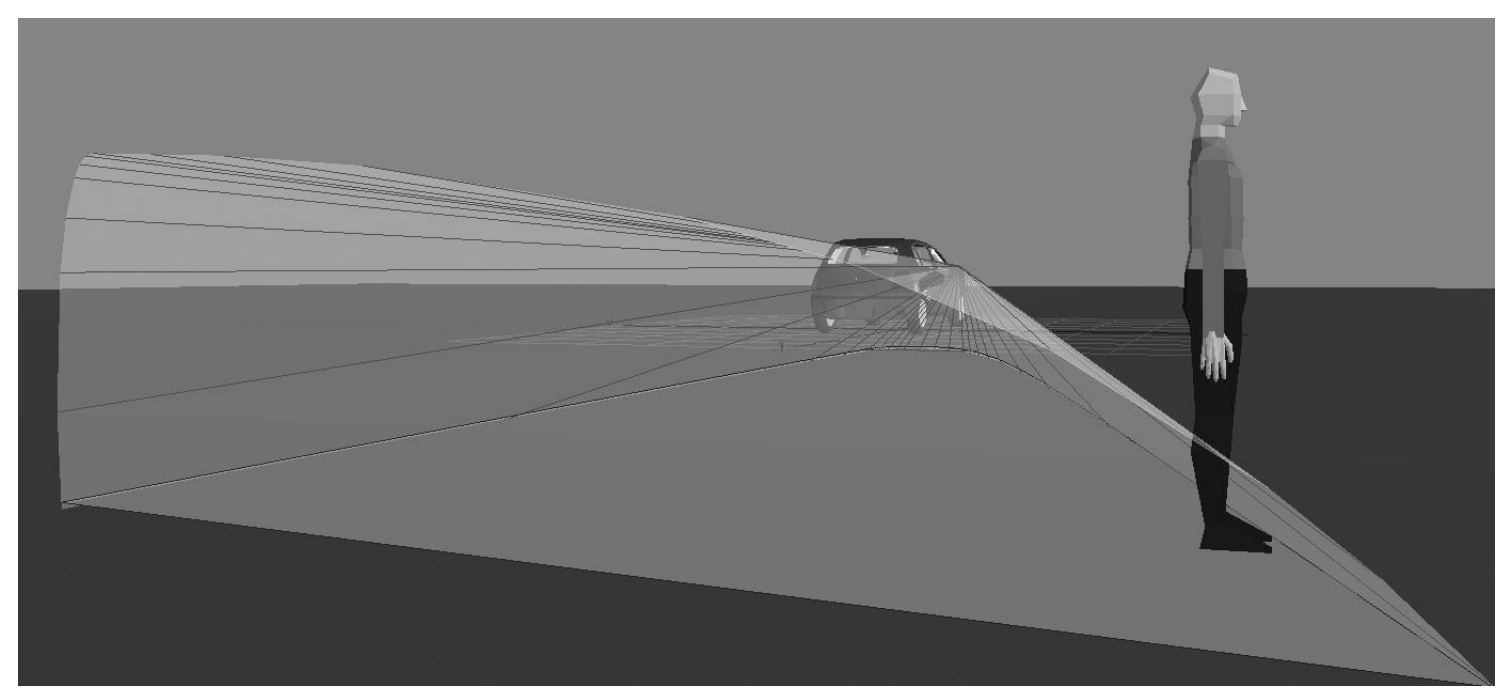

Figure 4. 3D volumetric field of view projection through a car's wing (side) mirror and the ability to view a target within the visible $2 \mathrm{D}$ area projected on the ground plane.

A final issue involves the combination of the respective areas of visibility and any blind spots. Taking a 2D approach can rarely capture the complexity of interaction 
of the visible volumes that occur in the 3D environment. In addition, evaluations tend to focus on one direction of view at a time and thus the interaction of multiple fields of view such as that shown in Figure 1 are not explicitly conveyed.

In order to address these issues the research produced a specification for a new tool to allow an improved understanding to be gained for field of view evaluations from vehicles. The tool should support:

- The 3D projection of the viewable volume as afforded the driver by direct vision through an aperture such as a window, or by indirect vision through a mirror.

- The projection of both flat and curved (spherical) mirrors.

- The viewable volume should be shown as a graphical object to be able to identify the interaction of this volume with objects in the virtual environment.

- Multiple apertures / mirrors should be able to be projected simultaneously to support a full $360^{\circ}$ evaluation of field of view around a vehicle.

- Individual projections should be distinguishable from one another.

- Projected volumes should be semi-transparent to allow a view to be taken of volume and its interaction with the environment.

- The volumes should support 2D clipping to the ground plane for comparison with existing regulations or previous work.

- $2 \mathrm{D}$ clipping should also be possible to any arbitrary 2D plane, not restricted to the ground plane.

- This tool should be implemented in a system that supports 3D modelling of environments and virtual, articulated humans to allow accurate modelling of driver eye locations. 


\section{Development of a 3D volumetric projection of field of view}

To address the requirements of the research and the specification a 3D volumetric field of view projection tool was developed and implemented within the existing SAMMIE DHM system. The development was based on an existing projection methodology within SAMMIE, similar to that found in other DHM systems such as Jack (Siemens PLM 2012) and RAMSIS (Human Solutions 2012), and other bespoke tools (Way and

Reed 2003). Working with an existing DHM system also provided the benefits of an existing modelling environment, an articulated and constrained human model and associated rendering tools.

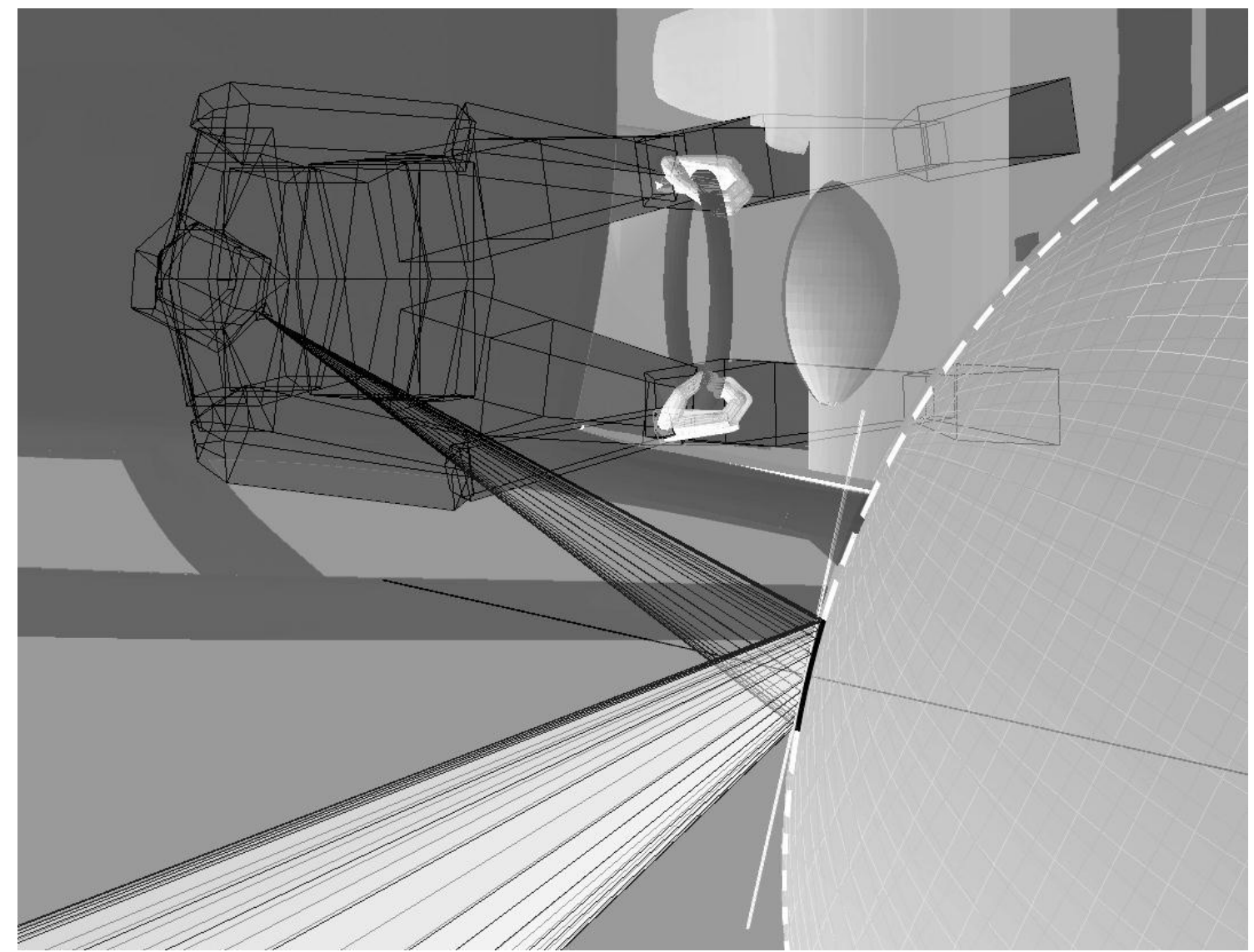

Figure 5. Schematic of the mirror projection technique. Rays extend from the driver's mean eye point and are reflected at each vertex point on the boundary of the spherical mirror surface. The image shows the virtual sphere from which the mirror surface is taken and the plane and normal of the mirror.

The core implementation of the projection tool involves the projection of a ray from the driver's eye point through each vertex of the polygonal window or mirror 
object and then tracing that ray to a user definable cutoff. The tracing of the projected ray is a straightforward continuation of the eye-vertex vector for aperture projection. For mirrors, the ray is reflected taking into account the curvature of the mirror. Curved mirrors are assumed to be spherical and must be defined together with their centre of curvature and radius of curvature. Figure 5 shows an illustration of the projection technique for a curved mirror. Rays extend from the driver's eye point (typically mean eye point) to each vertex on the mirror boundary. The mirror is defined by this series of boundary vertices, together with its centre of curvature (the centre of the sphere from which the mirror surface is 'cut'), and its radius of curvature (the radius of the sphere). The rays are then reflected and projected until they are trimmed to a user-definable cutoff.

Where this technique differs from other existing techniques is that the resulting projected rays are subsequently used to create polygons that together form a surface that encloses the visible volume of space. Each adjacent pair of rays together with the edge between the two vertices through which the rays have been projected, and the points resulting from the cutoff are used to form a four-sided polygon. These polygons are then rendered semi-transparently within the model to provide a visible volume that does not fully obscure other objects in the environment.

To support the need to be able to compare the $3 \mathrm{D}$ volumetric projections to $2 \mathrm{D}$ regulations and existing 2D plots, the field of view volume is also intersected with a user defined plane. The clipping results in a 2D area / boundary object created on the defined plane.

Finally, the projection routine was implemented to support up to 10 simultaneous mirror projections and 10 aperture projections. This enables the user to evaluate the projection of multiple windows and mirrors for a single vehicle to gain a 
full $360^{\circ}$ field of view assessment (Figure 6). Each projection has its own colour to aid differentiation.

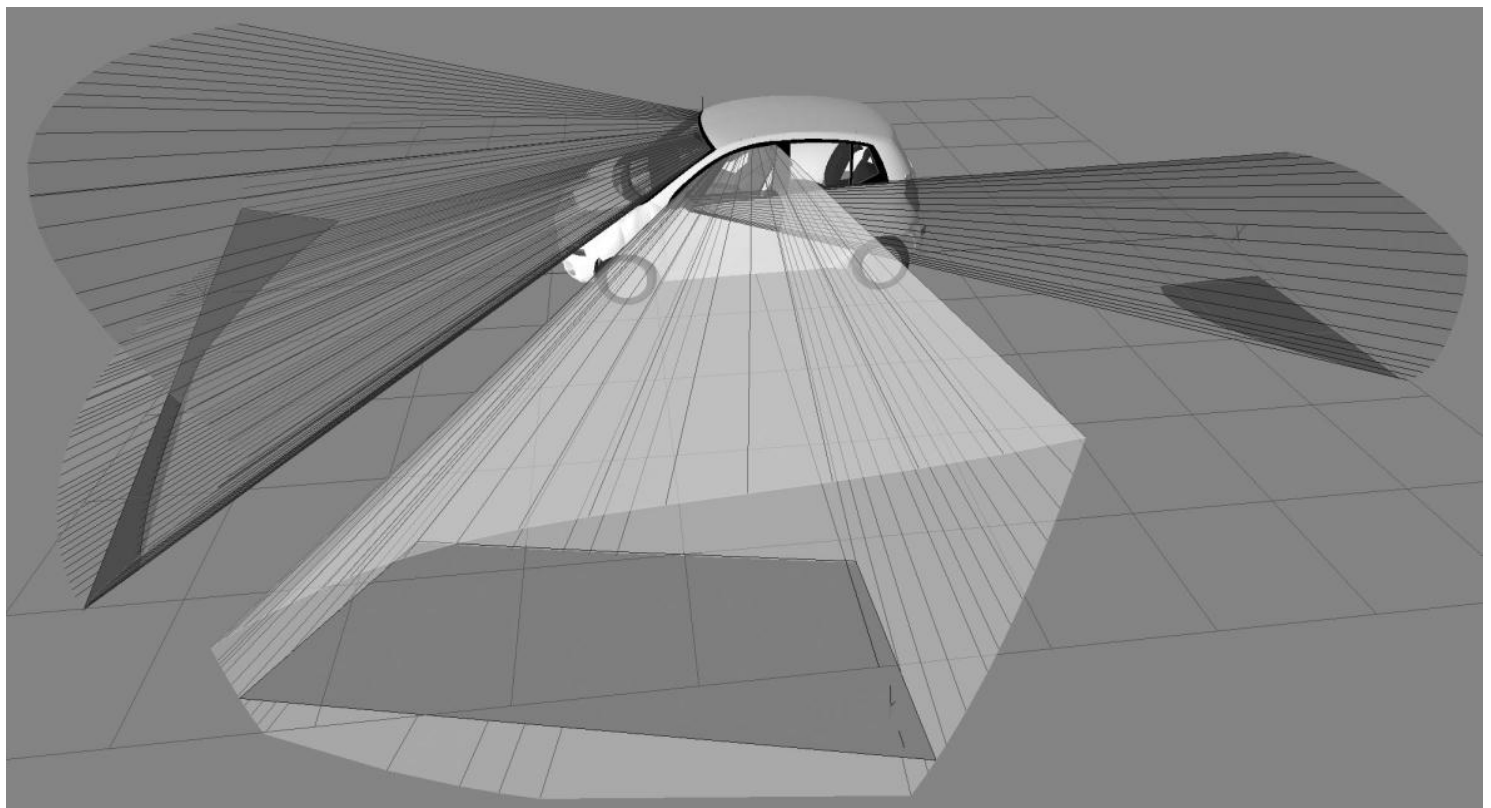

Figure 6. A multiple aperture volumetric projection (front window, left front window, left Class III mirror) on a 2010 model year Hyundai i10. The darker shaded areas indicate intersection with the ground plane.

\subsection{Validation of the $3 D$ volumetric projection}

The aim of the validation of the 3D volumetric projection was to understand if the projection in the digital environment was representative of the real world field of view. Due to the simplicity of the mathematical modelling of the projection of direct vision it was decided that the validation should focus on the projection of indirect field of view, through mirrors.

\subsubsection{Validation Methodology}

The methodology for the validation was to create a real world projection of an equivalent of the field of view visible through a mirror. As opposed to using human vision and the difficulties in quantifying whether a target marker is or is not visible in the mirror surface it was decided that a light source should be used to project visible light through the mirror, analogous to the techniques applied by other research in this 
area (Fosberry and Mills 1959). In addition, the recording of the projection should be done through the area projected on the ground plane to provide an easily quantifiable result. This approach relies upon the assumption that if the projected area is representative then the volume from which it is derived must also be representative as the volume is formed by vectors directly between the edge of the mirror surface and the projection area.

Table 1. Summary of Mirror Descriptions as specified in ECE46-02 (2009).

\begin{tabular}{|c|l|}
\hline Mirror Class & Purpose \\
\hline I & Interior rear-view mirrors \\
\hline II & Main exterior rear view mirror (large) \\
\hline III & Main exterior rear view mirror (small) \\
\hline IV & "Wide-angle" exterior mirror \\
\hline V & "Close-proximity" exterior mirror \\
\hline VI & Front mirror \\
\hline
\end{tabular}

The experimental setup can be seen in the left image of Figure 7. A mirror, a SPAFAX VM5 - Class V (see Table 1 for mirror classification), was clamped to a simple metal structure to suspend it at a known height above the floor, in this case $1000 \mathrm{~mm}$ to the centre of the horizontal pole. The mirror was clamped at a 45 degree angle to the floor. Below the mirror the floor was covered in $10 \mathrm{~mm}$ squared paper to form a large recording sheet. The light source was a Lenser T7 LED torch mounted to a tripod aligned with the approximate centre of the mirror surface $(900 \mathrm{~mm}$ high). The torch lens was located at a distance from the mirror surface equivalent to the distance between an LGV driver's eye point and the location of a Class V mirror (2m). The whole setup was located in a room without windows to ensure complete darkness. 


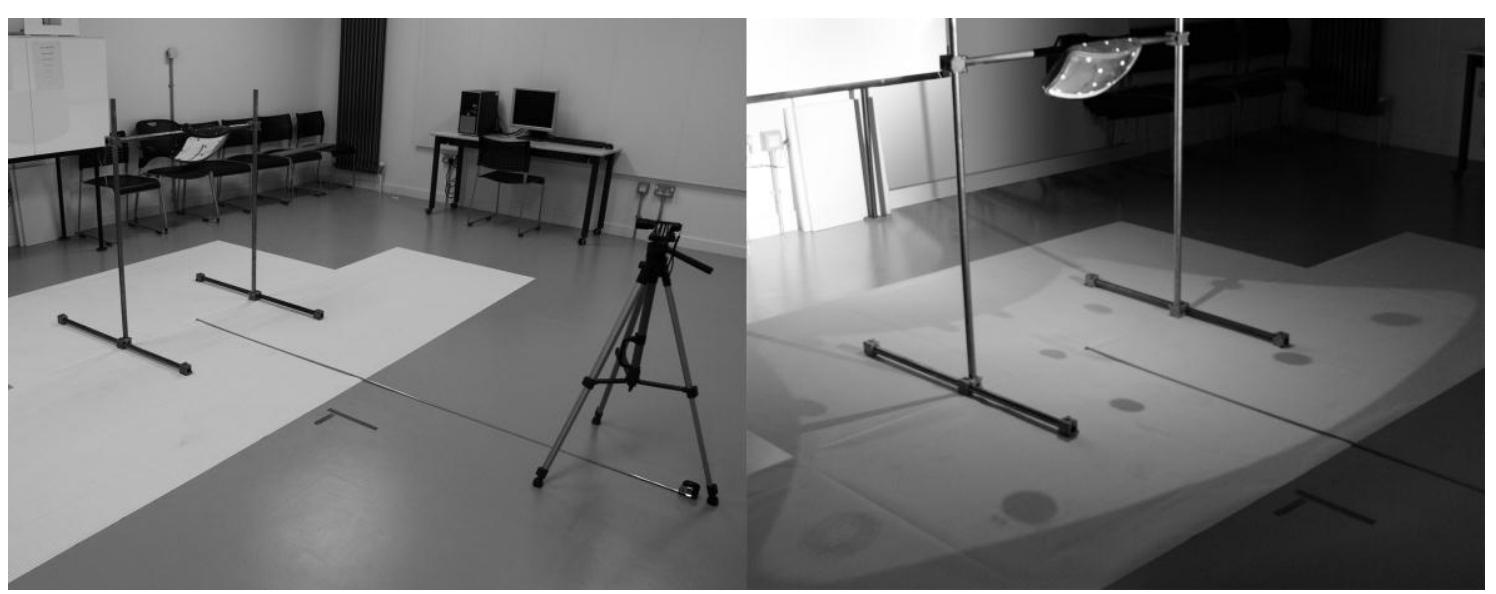

Figure 7. Validation of mirror projection, left image shows experimental setup, right image shows real world projection.

To allow modelling to take place the mirror surface and housing were 3D scanned using a ZCorp Zscanner 800 hand held laser scanner. The resulting can is essentially a series of points (point cloud) that lie on the surface of the scanned object. The mirror scan was imported into the CAD tool PRO/Engineer where surfaces were overlaid onto the points, and then finally the mirror object was imported into SAMMIE. The whole experimental setup was then recreated in the SAMMIE system with a human positioned with their eye-point to coincide with the recorded location of the light source.

\subsubsection{Validation Results}

The projection formed by the reflection of the light through the physical mirror in a darkened room was traced onto the squared recording paper on the floor (right image of Figure 7. The edge of the projection was not a perfectly defined line between the lit area and the non-lit area and so the perimeter of the projection must be considered with a tolerance of $\pm 5 \mathrm{~mm}$. The squared grid paper was then used to digitise the path that forms the boundary of the projected area into a series of coordinates. These coordinates were then used to recreate the path in the digital environment. With the model setup as described the digital projection was also created (right image of Figure 8) and cropped 
to the ground plane to form the equivalent projection area. The left image of Figure 8 shows the two resulting projections. The white line shows the real world projection path and the grey line the digital projection.

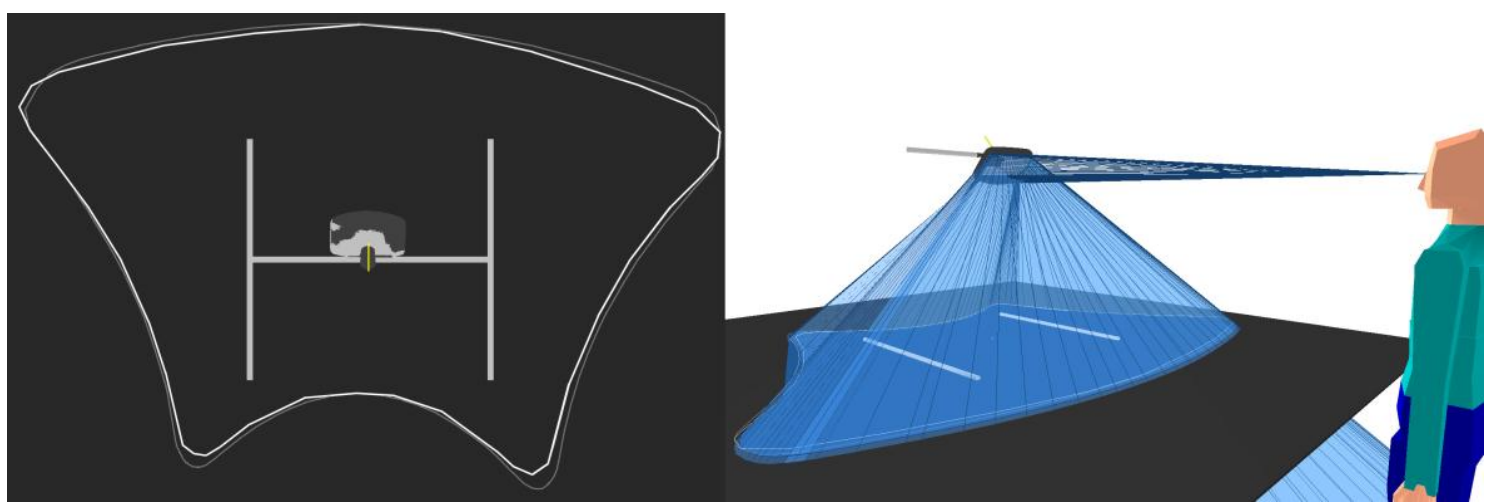

Figure 8. Validation of mirror projection, left image shows the two projected area boundary paths $($ white line $=$ real world, grey line $=$ digital), right image shows digital projection setup .

The 'shape' of the digital projection is equivalent to the real world projection with the same basic geometry and distinctive curved form. The geometrical difference in the projections is more pronounced at the corners and the digital projection is slightly larger.

The linear difference between the two paths was measured by projecting a ray from the centre point of the projected area outwards in increments of 10 degrees about the vertical axis. The linear distance between the centre point and the boundary path along each ray was measured for each projection and the difference calculated. From the 36 measurements the mean difference recorded was $24 \mathrm{~mm}$ to the nearest millimetre, and the maximum difference was $62 \mathrm{~mm}$.

\subsubsection{Validation Discussion}

The results of the validation have shown that there are differences between the real world and digital projections. As part of the process in understanding the differences a range of variables were explored. 
- Accuracy of recording the real world projected area. As previously discussed the boundary of the projection was not a crisp delineation and so the real world projection path is subject to a tolerance of approximately $\pm 5 \mathrm{~mm}$.

- The recording of the real world configuration. The angle of the mirror, captured with an inclinometer, the distance between the mirror and the torch, captured with a tape measure, and the use of the centre of the lens of the torch, are all subject to (relatively minor) inaccuracies in their recording. Of particular note is the equivalence of the digital projection that uses a single point in space from which all rays emanate and the torch bulb-reflector-lens configuration that likely results in the rays emanating from an area.

- The accuracy of the scanning process of the mirror to create a digital equivalent and in particular the capture of the boundary of the mirror surface. The scanner used has a reported accuracy of 40 microns in $\mathrm{X}$ and $\mathrm{Y}$ however this is for a single point and the points have to ultimately be surfaced to create the mirror. Due to the scanning process the surfacing is always an approximation of the real world object.

- Variation in the radius of curvature of the mirror due to manufacturing tolerances. The mirror used in the real world evaluation was measured three times by the manufacturer and a mean reading of $307 \mathrm{~mm}$ was reported. This value was used for the radius of curvature of the digital mirror. In the digital evaluation this results in a perfectly true sphere of consistent radius. In the real world the radius at any given point will vary form the mean. For context, the manufacturer reported that their polycarbonate mirror lenses have an allowable tolerance of $\pm 15 \%$. 
- The real world mirror has a lip formed from the plastic surround of the mirror that extends away from the mirror surface to a depth of approximately $2 \mathrm{~mm}$. The lip will occlude certain rays from the light source to the mirror and some rays after projection from the mirror surface. Thus the real world projection is likely to be affected when compared to the digital projection that does not include the lip. The lip is not modelled as the projection technique does not include the clash detection necessary to identify obstacles within the path of a projected ray.

To understand the potential impact of the variables identified, the validation explored the effects of some of these variables on the location of a single projection point. Table 2 shows the maximum difference recorded in the distance between the centre of the projected area and the relevant data point. From the observed differences it is clear than minor changes to the experimental, and therefore real world, configuration can result in changes up to approximately $100 \mathrm{~mm}$ in a point on the boundary of the projected area at a height of $1 \mathrm{~m}$. Through the control of many of the variables identified it is believed that the difference observed between the two projections is primarily due to the lip on the real world mirror that is not modelled in the digital environment.

Table 2. Maximum change is linear distance from the centre of an area projection to a single point on the boundary of the area due to a change in a number of potential variables.

\begin{tabular}{|l|l|}
\hline Variable & Deviation (mm) \\
\hline Change in radius of curvature of 1 degree (307 to 306mm) & 38 \\
\hline Change in eye point location (50mm closer to the mirror) & 7 \\
\hline Change in eye point location (50mm to the right) & 46 \\
\hline Change in eye point location (50mm up) & 51 \\
\hline $\begin{array}{l}\text { Change in mirror angle (from 45 degrees to the horizontal } \\
\text { to 44 degrees) }\end{array}$ & 116 \\
\hline
\end{tabular}

Taking all of the factors into account the results appear to show a representative projection is achieved through the digital tool with an acceptable level of variation. The 
differences in projection are well within the variation likely to be observed when the combined factors associated with eye point movement, mirror surface quality, and mirror setup are considered for any single viewing task in the real world.

\section{Case study - investigation of the rearward field of view for category $M_{1}$ and $M_{2}$ vehicles}

Once the projection tool had been developed and validated it was then implemented in the DfT field of view research project outlined in the introduction. A brief overview of one of the studies conducted is given here to illustrate some of the benefits and limitations of the technique.

As described in the introduction one particular element of the research aimed to address an ongoing concern within the DfT regarding rearwards field of view for category $\mathrm{M}_{1}$ and $\mathrm{M}_{2}$ vehicles. The specific concern was that vehicles may be able to comply with the standard for rearwards visibility but not actually provide an adequate field of view in close proximity to the vehicle. The evaluation consisted of the following stages:

(1) Two appropriate vehicles were identified, including: one current model (2010 on) Category $\mathrm{M}_{1}$ Volkswagen Touran, and one current model (2006 on)

Category $\mathrm{M}_{2}$ Ford Transit in long wheelbase mini-bus configuration (see Table 3 for basic dimensions).

(2) The capture of 3D data from these vehicles.

(3) CAD Modelling of the vehicles and the building of mirrors.

(4) The analysis of the CAD models to determine the rearward visibility from the vehicle. 
Table 3. Manufacturer supplied vehicle dimensions for the two evaluation vehicles.

\begin{tabular}{|l|l|l|l|}
\hline \multirow{2}{*}{ Vehicle } & Dimensions (mm) \\
\cline { 2 - 4 } & Length & Width & Height \\
\hline Volkswagen Touran (2010 on) & 4405 & 1795 & 1635 \\
\hline Ford Transit long wheelbase (2006 on) & 6403 & 2084 & 2385 \\
\hline
\end{tabular}

Both of the vehicles assessed in this project were recreated from the real vehicles as original CAD data were not available. At the MIRA vehicle testing facilities in Nuneaton, UK, a FaroArm mounted touch probe was used to trace contours on the vehicles to capture all of the major exterior surfaces and key interior elements (pillars and trim in proximity to apertures, seat contours at extremes of adjustability, steering wheel and adjustability etc.) as well as glazed apertures and mirror surfaces.

These data were then taken into the PRO/Engineer, curves were mapped onto the captured contours, and then surfaces created from the curves to model the various features of the vehicles. The resulting surfaces were then exported into SAMMIE. In SAMMIE the vehicle elements were named, grouped, and coloured appropriately to create realistic looking models. Where necessary, generic additional elements such as wheels, bumpers, lights, etc. were added to increase realism. Adjustability for mirrors, seats, headrests, and steering wheels were implemented as 'modifications' within SAMMIE to provide automated and constrained control over the positioning of those elements.

As part of the data capture process the SAE h-point manikin (SAE standards: J826 1995, J4002 2010) was used to establish a h-point reference for positioning the human models within the SAMMIE system. The procedure utilised the original h-point manikin and process outlined in J826 (1995). To aid posturing, the torso, hip and lower leg structure of the manikin was also scanned to allow a virtual equivalent of the h-point manikin to be oriented accurately within SAMMIE. 
To finalise the posture the driver is manipulated to provide a realistic driving posture, relatively reclined for the larger driver, relatively upright for the smaller driver. Posture was informed by evaluations of real drivers in the associated vehicles, particularly the LGV drivers evaluated elsewhere in the research project, and through the application of mean joint angle data from Porter and Gyi (1998). In addition, driver's view and the position of the steering wheel (if it is adjustable), to ensure a clear view of the road and of the instrument binnacle are used to help posture the human. Further posturing was done to provide an appropriate grip of the steering wheel and to position the right leg on the accelerator pedal (Figure 9).

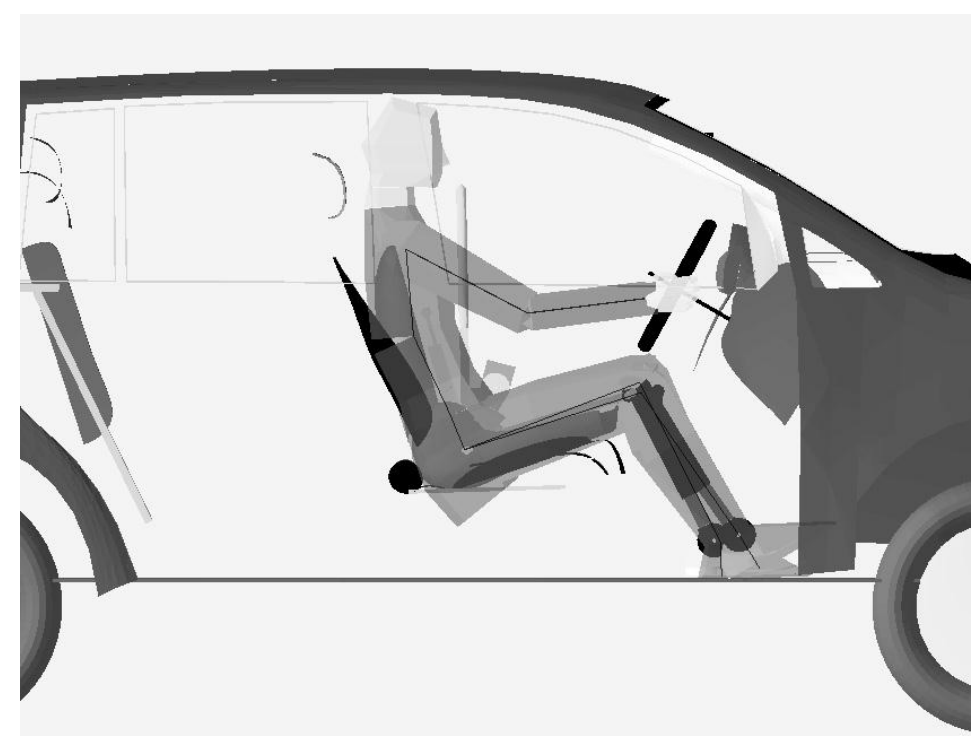

Figure 9. Vehicle image showing the driver's seat and the Dutch male driver positioned based on the virtual h-point manikin.

The evaluation methodology consisted of evaluating direct vision through the glazed areas of the vehicle together with indirect vision through the mirrors available on each vehicle. The Volkswagen Touran had Class I and III mirrors fitted, the Ford Transit had Class I, III and IV mirrors fitted. The evaluation was completed with two 'driver' human models selected to represent the extremes of the eye-point range. In order to determine these extremes the human models used consisted of both the largest and smallest people capable of being postured according to the procedure described 
above in a representative driving posture within the vehicles. This resulted in the use of three human models including a 99\%ile Dutch male (1941mm stature) for both vehicles to represent the largest driver, and a 5\% ile UK female (1534mm stature) for the Transit, and a $35 \%$ ile UK female ( $1615 \mathrm{~mm}$ stature) for the Touran to represent the smallest driver. All adult anthropometric data were taken from ADULTDATA (1998).

Rearwards visibility was assessed both ideally and in a real world sense that takes into account obscuration from passengers, interior fittings etc. The evaluation also included a number of assessments of indirect visibility for the relevant Classes of mirrors fitted to the vehicles compared of the field of view specified in standards ECE46-02 and 2003/97/EC.

\section{Results}

Due to the extensive nature of the evaluations performed a full treatment of results cannot be addressed here. The full results can be found in Cook et al. (2011). However, this section will focus on the use of the volumetric projection technique and how it performed in supporting the evaluations.

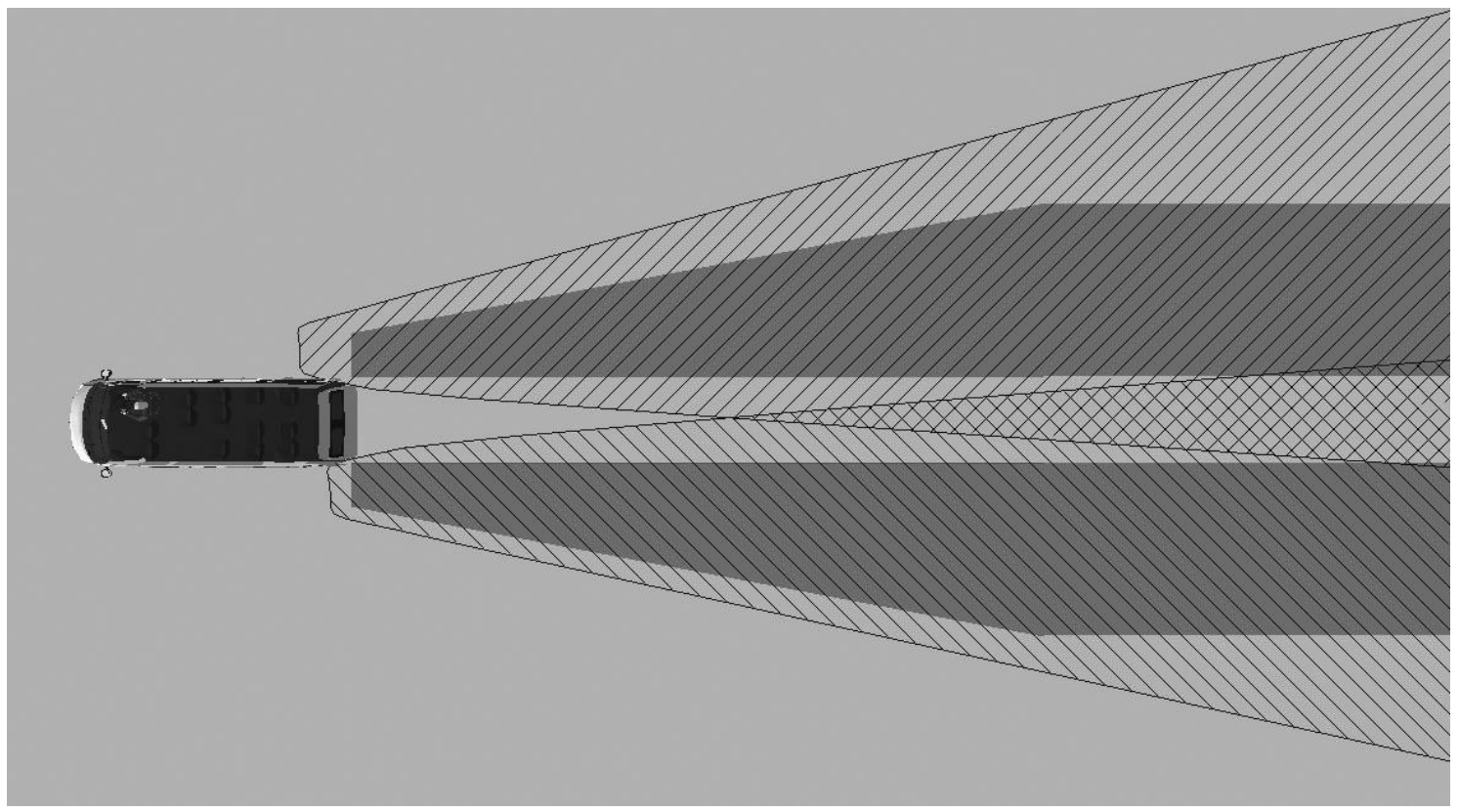

Figure 10. Indirect rearwards view and correlation with the minimum field of view specified in ECE46-02 and 2003/97/EC (Class III mirrors on the Ford Transit) 
Figure 10 shows how the 2D area clipping allows straightforward comparison between the indirect projected field of view from the Class III mirrors of the Ford Transit (hatched area) and the regulated areas (dark grey area, also see Figure 3). However, Figure 11 shows the power of the 3D volumetric analysis to provide a much better understanding of the field of view afforded to the driver. Here a child is standing adjacent to the vehicle in a position where they may be standing waiting to enter the passenger door. The child is $1220 \mathrm{~mm}$ in stature, which is representative of a 5\%ile $9 \mathrm{yr}$ old UK Male (source CHILDATA (1995)). This is an area clearly outside of the regulated area of visibility of the Class III mirrors. It is also an area that is not directly visible either through the door window apertures, or the windows in the side of the body of the vehicle. Figure 9 clearly shows the child stood between the projections from the side windows, however it also shows that the child's head is visible within the Class III projection volume. This can be confirmed by creating a 2D plot on a horizontal plane 970mm above the ground (the height of the child's neck) as shown by the hatched area in Figure 12.

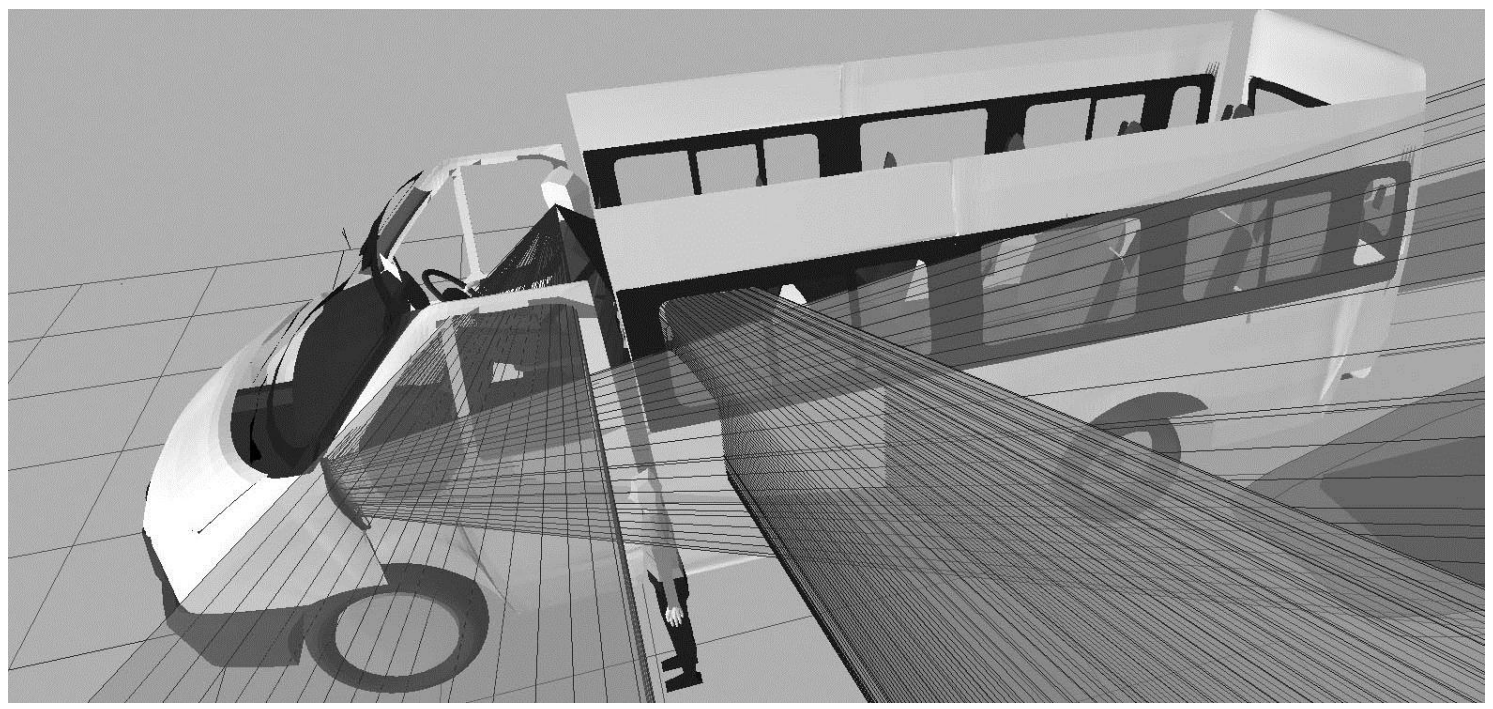

Figure 11. Volumetric projection analysis showing how the regulated area of visibility provides limited indication of the field of view afforded to the driver. 5\% ile 9yr old UK Male $(1220 \mathrm{~mm}$ stature) standing adjacent to the vehicle. 


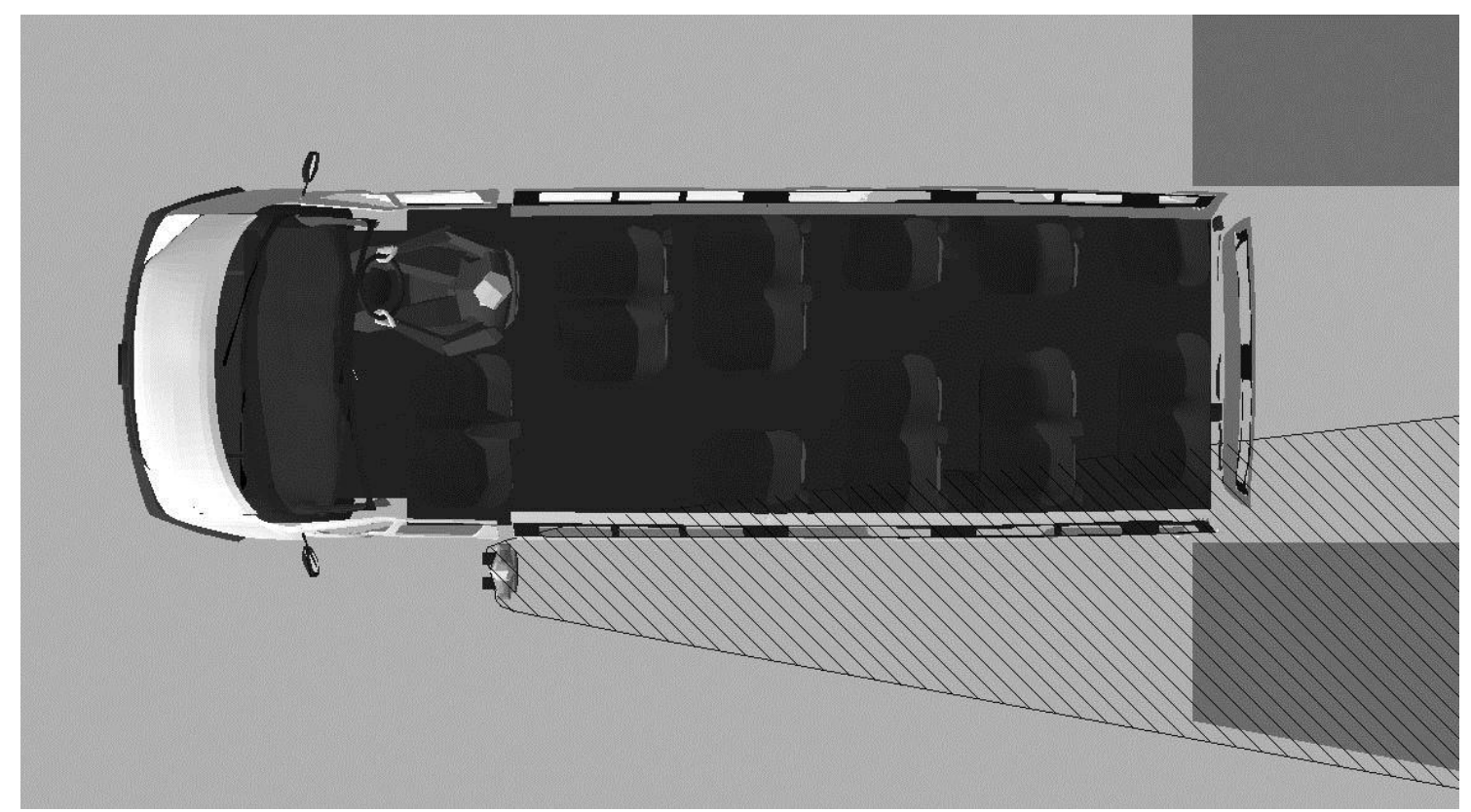

Figure 12. Indirect rearwards view from the left Class III mirror on the Ford Transit projected onto a plane parallel to the ground at $+970 \mathrm{~mm}$.

Figure 13 shows the projected areas for both the Ford Transit and the Volkswagen Touran for Class I mirrors. The ground plots indicate the presence of a significant blind spot directly rearwards of both vehicles. In both instances direct vision (white hatched areas) affords a greater viewable area but both meet the necessary regulation for field of view in the Class I mirror. Again the volumetric approach adds significantly more information and insight to these 2D area plots. Figure 14 shows the maximum height of person that can be obscured directly rearwards of the vehicle, in this case a 5\%ile 14yr old UK Male (1400mm stature, source CHILDATA (1995)).

Alternatively this could be interpreted that someone would need to be taller than $1400 \mathrm{~mm}$ to have any possibility of being visible to the driver. The right hand image in Figure 14 also shows that the view from the driver's eyes indicates that the visibility may be even more compromised due to internal fixtures such as the head restraints. Whilst not shown in the figure, the presence of passengers would exacerbate this problem further. Though not directly detailed here, the research did explore this issue and found that the presence of passengers could result in the rearwards view through 
Class I mirrors and via direct vision, in both vehicles, being effectively obscured to the driver.

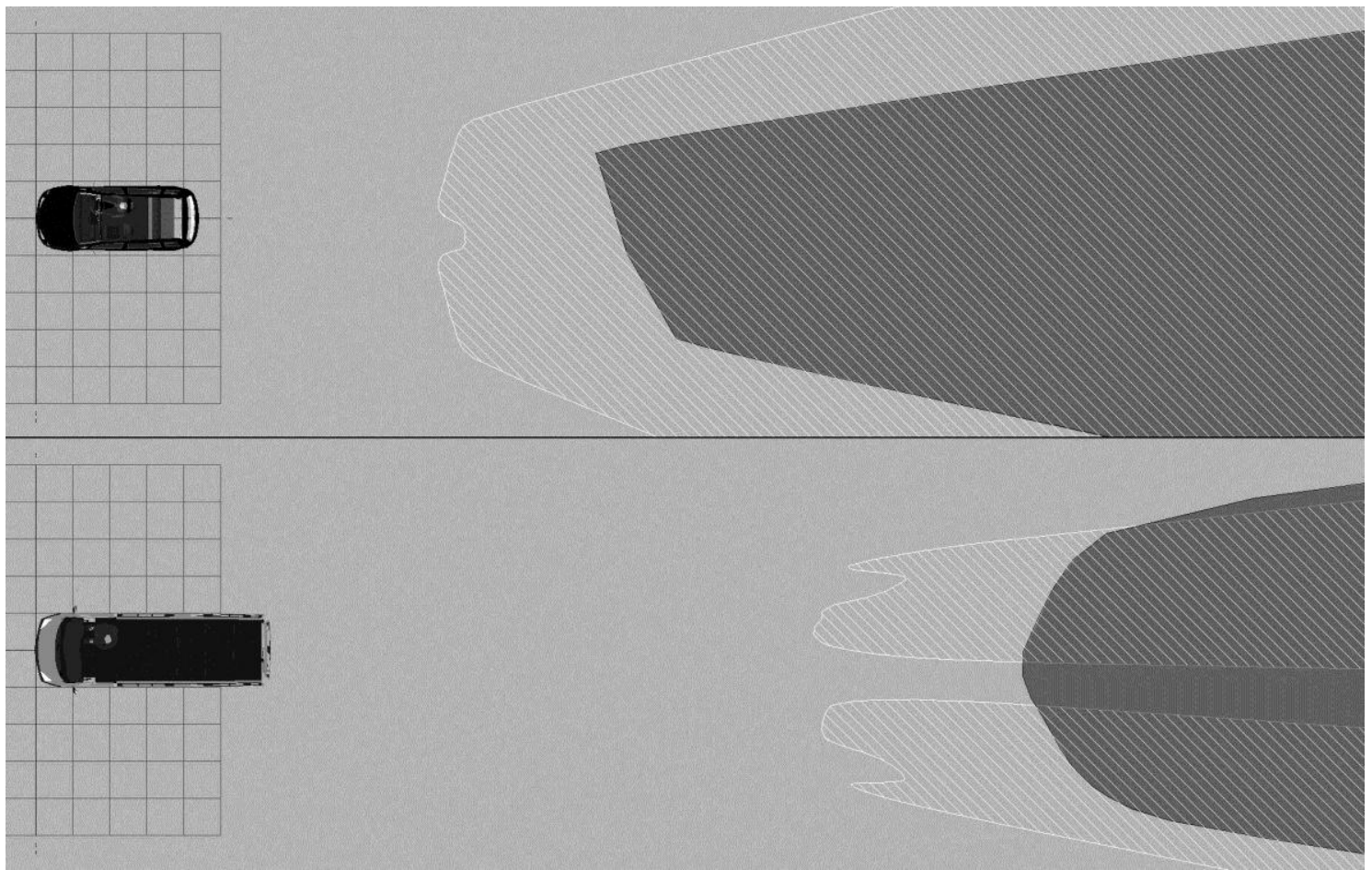

Figure 13. Direct (Rear window(s) - white ) and indirect (Class I mirror - black) rearwards view for the Volkwagen Touran (top) and Ford Transit (Bottom). Viewable areas shown for the 99th \%ile Dutch male (1946mm stature).

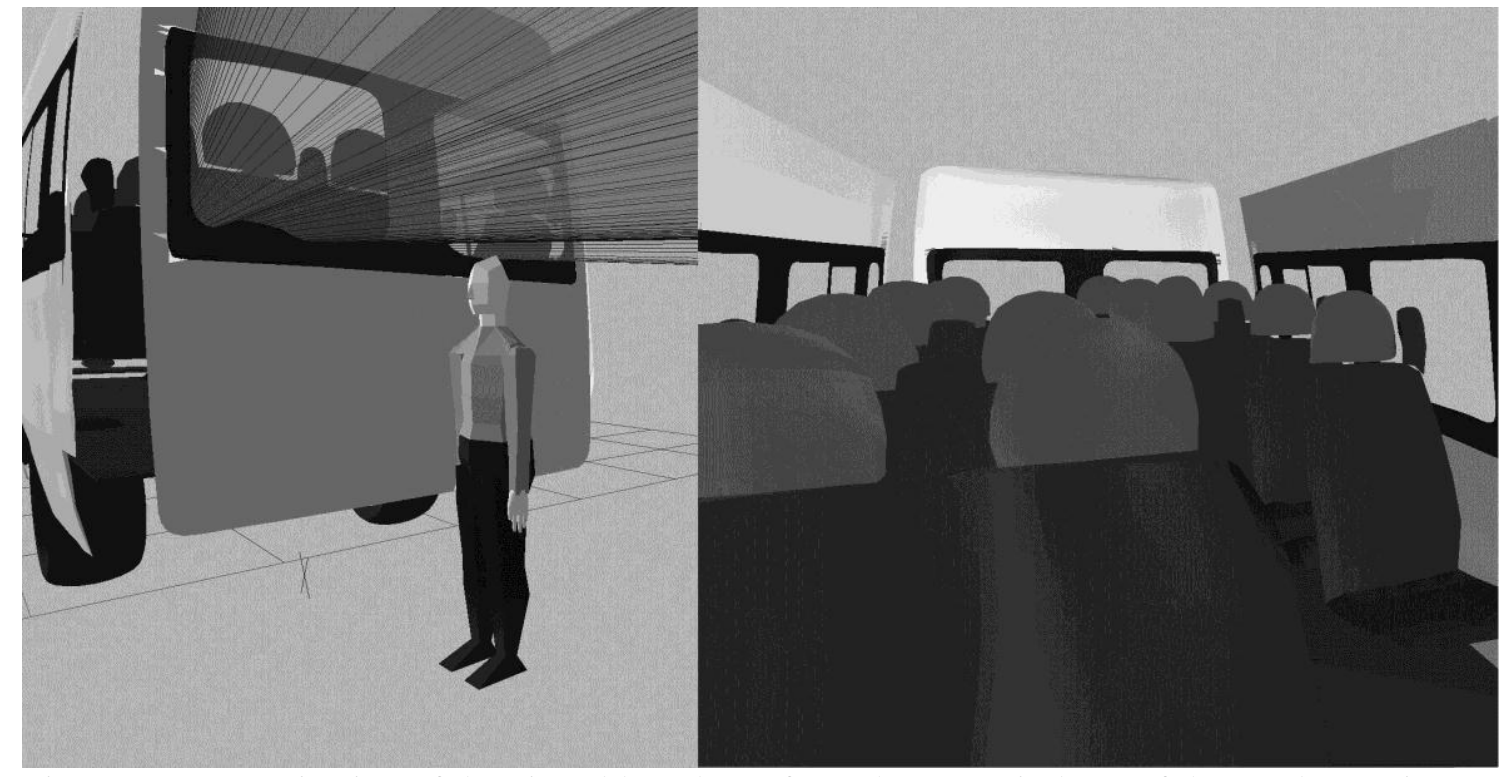

Figure 14. 3D projection of the viewable volume from the rear windows of the Ford Transit (left) and the view of the driver looking rearwards (right). 5\% ile 14yr old UK Male $(1400 \mathrm{~mm}$ stature) standing rearwards of the vehicle. 
In summary, the analysis showed that both vehicles complied with the regulations for Class I and III mirrors. However it also highlighted that there was a clear blindspot for both vehicles directly rearwards of the vehicle. The smallest blind spot as experienced by the larger driver in the Volkswagen Touran showed that an area up to $6.5 \mathrm{~m}$ rearwards of the vehicle on the ground plane or $0.5 \mathrm{~m}$ on a plane $1 \mathrm{~m}$ above the ground were not visible. Clearly this highlights the potential for a small child, or child seated on bicycle, or on the floor, to be easily hidden from the driver's field of view.

\section{Discussion}

The volumetric approach, used to augment the 2D regulated areas of visibility, proved to be extremely useful in gaining a much better understanding of the visibility afforded to the driver and a means to evaluate different scenarios in which accidents could occur.

Solutions to the issue of rearwards visibility that result in the driver being able to see the occluded area are not straightforward. It is recognised that the driver is unlikely to be able to see the area directly rearwards of the vehicle in any easily implemented manner. Thus the recommendation is that the driver is aided in detecting an obstruction within the blind spot through a non-visual method. The primary recommendation of this particular area of the research project was that the effective blind spot rearwards of these categories of vehicles is addressed through already existing rear detector systems. These systems are increasingly common on $\mathrm{M}_{1}$ vehicles either as standard fit, an additional option, or as a retro-fit option. The technology is mature and their function appears to be robust, in addition their cost is relatively modest.

For vehicles with essentially zero direct rearwards field of view such as the $\mathrm{M}_{2}$ vehicle evaluated these should be considered to be mandatory to afford some rearwards coverage. For $\mathrm{M}_{1}$ vehicles, mandatory fit may be less clear with the prevalence of 
serious accidents needing to be evaluated against the ability for the technology to have prevented the accident and the ultimate cost of forcing manufacturers to fit this technology. It may be true that this technology will be sufficiently ubiquitous in a few years' time to be considered standard equipment on new vehicles. However, reversing accidents are still clearly a major concern as illustrated by the RoSPA (2011) report on 'Children In and Around Cars' that highlights the problems with reversing off driveways and the potential for accidents to occur to small children not visible to the driver.

During the evaluations performed in the research it was confirmed that the flexibility designed into the projection tool provides a number of useful features for the analysis of field of view. Taking a full 3D approach through the projection of volumes allows a much greater understanding of what can be seen by the driver. This is particularly important in complex scenarios where the driver may have a number of different apertures and mirrors through which they can see at any given point. In addition the volumetric projection provides a very powerful visualisation tool to support communication of the field of view to other stakeholders.

Validation of the 3D projection technique showed that the digital projection was cable of giving a good representation of real world projection. The validation also identified the potential variation within the field of view problem. Given the ability for a driver to change the field of view afforded them by merely moving their head the results from the validation are deemed to be of sufficient accuracy to provide the required insight into the issues of field of view coverage.

The 3D approach also highlights the limitations of the 2D areas mandated in the regulations. Whilst it is likely that the $2 \mathrm{D}$ areas have been designed with the $3 \mathrm{D}$ volume in mind this subtlety is lost to those looking to implement and abide by the regulations. 
Through the use of scenario testing such as that shown in Figure 10, it is clear that a 2D approach can introduce significant ambiguity into field of view analysis. Targets placed within a $2 \mathrm{D}$ field of view boundary may not actually be visible to any recognisable degree (Figure 4) and conversely targets that fall outside of the 2D boundary may actually be visible (Figures $9 \& 10$ ). Without a full understanding of how the $2 \mathrm{D}$ areas are determined it is almost impossible to fully understand the field of view afforded the driver. The use of the ground plane is the main concern in this approach. Almost all obstacles that a driver needs to be aware of have a vertical dimension and a proportion of this dimension needs to be visible to the driver to aid recognition. Being able to only see the soles of the shoes of a pedestrian or the bottom of a tyre of a road vehicle is unlikely to be sufficient for a driver to actually perceive an obstacle and yet technically they may be within the projected area. A simple step to aid in understanding the problem is the projection of $2 \mathrm{D}$ areas at different heights. This was done for all vehicles assessed in this research and does provide greater understanding. However the move to a 3D volumetric approach provides even greater understanding. A particular benefit is the ability to assess and understand the integration of the fields of view. If correctly specified field of view through direct vision and through indirect vision can be aligned to ensure appropriate coverage of the driving environment. However, conveying this interaction is very difficult in 2D. The evaluation of field of view is essentially as much a qualitative problem as a quantitative one and so the ability to visualise, dynamically, how much of a potential obstacle is visible to the driver, and to visualise the scenarios in which blindspots may become a real issue is particularly powerful, and only possible in a 3D environment.

However, the 3D volumetric projection technique implemented is not without its own limitations. The projection is still an 'ideal' projection. The projection cannot 
account for obstacles or obscurations to the field of view beyond the projected aperture / mirror. Thus the projection of the Class I mirror is particularly problematic as the projection does not account for headrests, other passengers etc. This was highlighted by the analysis of the Ford Transit that in an ideal situation complied with the field of view regulation for a Class I mirror, but in reality the view rearwards in the occupied minibus is essentially zero. Furthermore no account is taken of the ability to recognise targets within the volume. For indirect vision, target recognition is a significant issue. The use of non-planar (curved) mirrors introduces distortion. The distortion is more pronounced as the radius of curvature decreases. Thus the specification of mirrors is always a balance between their coverage and their distortion of the view. In addition to distortion due to their form, mirror views are also subject to distortion from the material of manufacture (driver feedback suggests that polymer mirrors are less clear than glass mirrors), from dirt and from rain, ice, or snow. Regular cleaning and heated mirrors can reduce the likelihood of some of these issues. However, real world testing of target recognition that was performed as part of this research, but is not detailed here, suggests that anything at the boundaries of the volume should not be relied upon to be recognisable and hence visible. Finally, the 3D approach is also limited by what makes it so powerful. The 3-dimensionality of the environment being modelled ideally requires a $3 \mathrm{D}$ medium for communication. Distilling the $3 \mathrm{D}$ environment into $2 \mathrm{D}$ images as illustrated in the figures does diminish their impact and understanding. Further work in this area should explore the potential for using more advanced ray-tracing techniques to allow the impact of obscuration within the projected volume to be considered in an objective manner. The use of ray-tracing has the potential to account for all incidental obscuration due to internal fittings (e.g. headrests), passengers, the mirror lip issue presented earlier, and concerns such as mirror views being obscured 
by other mirrors as was observed in some of the LGVs evaluated elsewhere in the research. Ray tracing of the volume could also be combined with ray tracing of the projected image providing an indication of the view afforded the driver on the mirror surface and an indication of the impact of distortion.

These technologies are readily available but currently only in disparate tools. Their combination in a DHM system would provide the ability to bring together CAD, human modelling, assessment and visualisation capability in one system. Ray-tracing of the projection, taking into account the complex environment, would provide clear mapping of all blind spots not only those caused by fundamental structure of the vehicle. These developments would support a much more objective review of the regulations in light of real-world usage of mirrors and direct vision to determine if the field of view was acceptable for the given task.

\section{Conclusion}

The UK Department for Transport have on-going concerns with driver visibility from vehicles and the role of driver blind spots in accidents. As part of a large, multidisciplinary project funded by the DfT, [a UK research institution removed for anonymity purposes] evaluated driver visibility and blind spots in Category $\mathrm{M}_{1}, \mathrm{M}_{2}, \mathrm{~N}_{2}$ and $\mathrm{N}_{3}$ vehicles. In order to support this evaluation a new 3D volumetric projection technique was developed and implemented in the existing DHM system SAMMIE. This volumetric technique allows the projection of the visible volume of space directly through window apertures, or indirectly through mirrors. In addition $2 \mathrm{D}$ area plots can be produced on any plane to support direct comparison with regulatory standards. The projection technique was subsequently validated through comparison to the projection observed using a mirror and a light source in a real world experimental setup. Through the use of the 3D volume projections the research identified a number of blind spot 
issues with vehicles currently on UK roads. The illustrative nature of the projections allowed a greater insight to be gained into the vision problems presented to the driver, and how they could act as a contributory factor in accidents. This paper shows how the technique was applied specifically to rearwards visibility for Category $\mathrm{M}_{1}$ and $\mathrm{M}_{2}$ vehicles and highlights the significant blind spot, directly rearwards of these vehicles. The research recommended that further research be carried out and that non-visual detector systems be mandated for Category $\mathrm{M}_{2}$ vehicles.

\section{References}

2003/97/EC. 2004. Directive 2003/97/EC of the European Parliament and of the Council on the approximation of the laws of the Member States relating to the type-approval of devices for indirect vision and of vehicles equipped with these devices. Official Journal of the European Union.

ADULTDATA. 1998. The handbook of adult anthropometry and strength measurements - data for design safety. eds. L. Peebles and B. Norris. Department of Trade and Industry.

Case, K., J.M. Porter, and M.C. Bonney. 1980. Design of mirror systems for commercial vehicles. Applied Ergonomics, 11.4, 199-206.

CHILDATA. 1995. The handbook of child measurements and capabilities - data for design safety. eds. B. Norris and J. R. Wilson. Department of Trade and Industry.

Cook, S. E., S. Summerskill, R. Marshall, J.H. Richardson, C. Lawton, R. Grant, S.H. Bayer, J. Lenard, and K. Clemo. 2011. The development of improvements to drivers' direct and indirect vision from vehicles. Phase 2. Loughborough University. Report for Department for Transport.

Dodd, M. 2009. Follow on study to the heavy goods vehicle blind spot modelling and reconstruction trial. Transport Research Laboratory: Published report PPR403. ECE46-02. 2009. Uniform Provisions for Devices for Indirect Vision and of Motor Vehicles with Regard to the Installation of these Devices. UNECE Vehicle Regulations. 
Fitch, G., M. Blanco, M.C. Camden, R.L. Olson, J. McClafferty, J.F. Morgan, A.E. Wharton, H.E. Howard, T. Trimble, and R.J. Hanowski. 2011. Field Demonstration of Heavy Vehicle Camera/Video Imaging Systems. SAE International Journal of Commercial Vehicles, 4(1) 171-184.

Fosberry, R.A.C. and B. C. Mills. 1959. Measurement of Driver Visibility and its Application to a Visibility Standard. Proceedings of the Institution of Mechanical Engineers: Automobile Division January 1959 13: 50-81.

Gruber, N. U. P. Mosimann, R. M. Mu“ri, and T. Nef. 2012. Vision and Night Driving Abilities of Elderly Drivers, Traffic Injury Prevention, In Press. DOI:10.1080/15389588.2012.727510

Hahnel, U.J.J. and Hecht, H. 2012. The impact of rear-view mirror distance and curvature on judgements relevant to road safety, Ergonomics, 55:1, 23-36.

Human Solutions., 2012. RAMSIS Automotive [online]. Available from: http://www.human-solutions.com/mobility/front_content.php?idcat=252 [Accessed 17 July 2012].

Krum, A., 2009. Visibility Modeling from a Heavy-Vehicle OEM's Perspective. Proceedings of the 19th Biennial TRB Visibility Symposium, May $12-14$, Virginia Tech Transportation Institute, 1-54.

Mansfield, H. A. Bunting, M. Martens, and R. van der Horst. 2008. Analysis of the On the Spot (OTS) Road Accident Database. Road Safety Research Report No. 80. Department for Transport: London.

Molinero, A. H. Evdorides, C. Naing, A. Kirk, J. Tecl, J.M. Barrios, M.C. Simon, V. Phan, and T. Hermitte. 2008. Accident causation and pre-accidental driving situations. Part 3. Summary Report. Deliverable D 2.3 of the Traffic Accident Causation in Europe project.

NHTSA., 2008. National motor vehicle crash causation survey, report to congress. US Department for Transport, National Highway Traffic Safety Administration. DOT HS 811059.

Porter, J.M. and Gyi, D.E., 1998. Exploring the optimum posture for driving comfort. International Journal of Vehicle Design 19 (3), 255-266.

Porter, J. M., R. Marshall, M. Freer, and K. Case. 2004. SAMMIE: A Computer Aided Ergonomics Design Tool. In Working Postures and Movements, Tools for Evaluation and Engineering, eds. Delleman, N, Haslegrave, C, Chaffin, D. Boca Raton: CRC Press. 454-470. 
Reed, M.P., Lehto, M.M. and Flannaghan M.J., 2000. Field of view in passenger car mirrors. The University of Michigan, Transportation Research Institute, Report No. UMTRI-2000-23.

RoSPA., 2011. Children In and Around Cars - Final Report. Royal Society for the Prevention of Accidents.

SAE J826., 1995. Society of Automotive Engineers, Devices for use in defining and measuring vehicle seating accommodation. SAE Standard, Vehicle Occupant Restraint Systems and Components.

SAE J4002., 2010. Society of Automotive Engineers, H-Point Machine (HPM-II) Specifications and procedure for H-point Determination - Auditing Vehicle Seats. SAE Standard, Vehicle Occupant Restraint Systems and Components. Siemens PLM., 2012. Jack and Process Simulate Human [online]. Available from: http://www.plm.automation.siemens.com/en_us/products/tecnomatix/assembly_ planning/jack/\#lightview-close [Accessed 17 July 2012].

Tait, A.R. and D. Southall., 1998. Drivers' field of view from large vehicles - Phase 3 Report. Loughborough University. Report for Department for Transport.

Teizer J., et al., 2010. Automating the blind spot measurement of construction equipment. Automation in Construction 19 (2010) 491-501.

Toet, A., S.E.M. Jansen, and N.J. Delleman. 2008. Effects of field-of-view restriction on manoeuvring in a 3-D environment, Ergonomics, 51:3, 385-394.

UNECE Regulation 46., 2009. Uniform Provisions for Devices for Indirect Vision and of Motor Vehicles with Regard to the Installation of these Devices. UN ECE Vehicle Regulations.

UNECE Regulations 41-60., 2012. UNECE Vehicle Regulations - 1958 Agreement, Addenda to the 1958 Agreement (Regulations 41 - 60) [online]. Available from: http://www.unece.org/trans/main/wp29/wp29regs41-60.html [Accessed 17 July 2012].

Way, M.L. and Reed, M.P., 2003. A Method for Measuring the Field of View in Vehicle Mirrors. Proceedings of the SAE 2003 World Congress, SAE Paper No 2003-01-0297, Detroit, USA, March 2003, 1-8. 